\title{
Displacement Back Analysis Based on GA-BP and PSO-BP Neural Network
}

\author{
Dongdong $\mathrm{Gu}^{1,2, \text { a }}$, Yunliang Tan ${ }^{1,2, \mathrm{~b}}$ \\ ${ }^{1}$ State Key Laboratory of Mining Disaster Prevention and Control Co-founded by Shandong Province and the Ministry of Science \\ and Technology, Shandong University of Science and Technology, Qingdao 266590, China; \\ ${ }^{2}$ College of Mining and Safety Engineering, Shandong University of Science and Technology, Qingdao 266590, China \\ aemail: gudong900@163.com, bemail: yunliangtan@163.com
}

\begin{abstract}
In order to study the back analysis accuracy of different algorithms of neural network for back analysis of tunnels surrounding rock. Firstly, the main parameters influencing the deformation of tunnels surrounding rock are analyzed by orthogonal test. Secondly, a FLAC3D numerical model of the tunnels was established based on the field conditions for getting learning samples of the neural work. Thirdly, the BP neural network is optimized by genetic algorithm, particle swarm optimization and normalization method, respectively. At last, the back analysis of the tunnels displacement is carried out, and the mechanical parameters of the surrounding rock are forecasted for comparing accuracy of the three optimized methods. The results show that it is faster and precise to use artificial neural network to inverse mechanical parameters of the tunnels surrounding rock.
\end{abstract}

Keywords - back analysis of displacements, BP neural network, genetic algorithm, particle swarm optimization

\section{INTRODUCTION}

Surrounding rock conditions are complex, such developed joint fissures, great lithology differences of roof, floor and two sides, etc. Coupling relationship exists among the deformation of roof, floor and two sides, and the damage of each section has a tight connection [1]. There are some discrepancies in the mechanics parameters acquired by the laboratory test and field test. The rock mass mechanics parameters can be got through the back analysis of the field monitoring displacement of the tunnels[2]. In recent years, Artificial Neural Network (ANN) proposed on the basis of modern neuroscience promotes the development of back analysis. It can imitate the functional characteristics of human brain, such as learning, classifying, and adjusting, etc [3]. The complicated nonlinear mapping relation between the displacement and mechanics parameters of the tunnels can be established based on the displacement back-analysis of the tunnels [4]. Meanwhile, the displacement of roof, floor and two side walls can be analyzed inversely. It is of practical significance for the tunnels support design.

The displacement of tunnels is mostly used the methods of Back-Propagation neural network (BP), genetic algorithm (GA) and radial basis function neural network. For example, Zhao et al [5] developed the program of elastoplastic displacement back analysis of the tunnels through combining the genetic algorithm and FLAC numerical software, and it can back-analyze parameters, such as $E, \mu, C, \varphi, \sigma_{\mathrm{x}}, \sigma_{\mathrm{y}}, \tau_{\mathrm{xy}}$. Wang et al [6] utilized the data of displacement of tunnels by finite element analysis as the training and testing samples to back-analyze displacement of the tunnels using BP neural network with particle swarm optimization (PSO). In this paper, back-analysis models are established using three different ANN methods, including BP neural network, the BP neural network optimized by the genetic algorithm (GA-BP) and the BP neural network with particle swarm optimization (PSO-BP), to back-analyze the rock mechanics parameters based on the displacement of the tunnels.

\section{CONSTRUCTION OF THE ANNS}

\subsection{Back-propagation neural network}

BP has fault tolerance and generalization capability. However, the BP can easily fall into local minimum and over fitting, which influence the predicting accuracy and reliability [7]. This paper optimizes the BP by Levenberg-Marquardt algorithm, sigmoid activation function and normalization method.

\subsubsection{The basic structure of BP neural network}

In this model, the displacement of roof, floor and two side walls of the tunnels are input parameters and three groups of elastic modulus and poisson's ratio are output ones. Meanwhile, all the datas will be normalized firstly to decrease the error introduced by different magnitudes. The number of hidden-layer nodes can be obtained from the Eq. (1) [8]. Thus, the $\mathrm{BP}$ architecture used to back-analysis of the displacement of the tunnels is 4-9-6.

$$
l=\sqrt{m+n}+a
$$

Where, 1 is the node number of implicit layer; $m$ is the node number of input layer; $n$ is the node number of output layer; $\mathrm{a}$ is the constant between $(0,10)$.

The topological structure of the BP used to back analyze the displacement of the tunnels is shown in Fig. 1. The chosen neural network architecture consistes of the input layer, the 
hidden layer and the output layer. $X_{1}, X_{2}, X_{3}, X_{4}$ are the input values and $E_{1}, \mu_{1}, E_{2}, \mu_{2}, E_{3}, \mu_{3}$ are the output values. $W_{\mathrm{ij}}(\mathrm{i}=1$, $2,3,4 ; \mathrm{j}=1,2, \ldots, 9)$ and $W_{\mathrm{jk}}(\mathrm{j}=1,2, \ldots, 9 ; \mathrm{k}=1,2, \ldots, 6)$ are the weight values of neural networks.

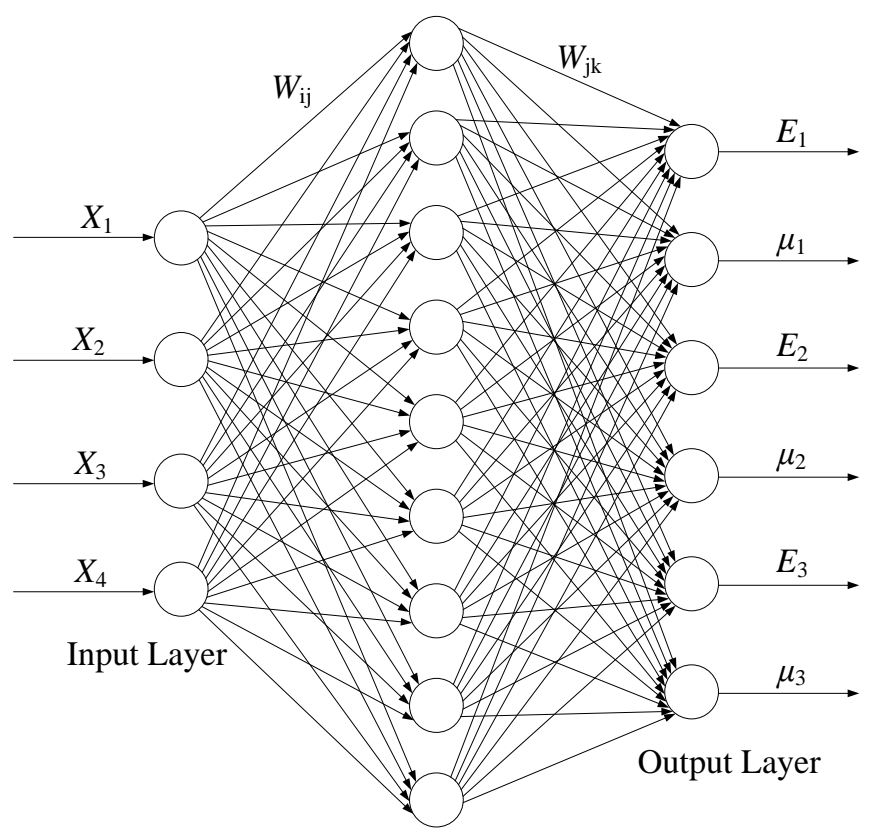

Hidden Layer

Fig.1. The topological structure of the BP neural network

\subsubsection{The activate function of BP neural network}

The relationship between the input values $\mathrm{x}_{\mathrm{i}}$ and the output values $\mathrm{x}_{\mathrm{j}}$ are shown in Eq. (2).

$$
x_{j}=f\left(\sum_{i=1}^{n} w_{i j} x_{i}+b_{j}\right)
$$

where, $W_{\mathrm{ij}}$ is the weight value between the node number of input layer $i$ and the node number of output layer $j ; b_{\mathrm{j}}$ is the threshold of the node $j ; f$ is the activation function.

In this paper, Hyperbolic Tangent function is selected as activation function. It can approximately nonlinear relationship between inputs and output, so that the network has better fault tolerance. Another important feature is that the activation function is continuously differentiable, which means it can be calculated by using the gradient method strictly.

The function expression and the image are shown in formula (3) and Fig.2, respectively.

$$
f(x)=\frac{2}{1+e^{-2 x}}-1
$$

\subsubsection{The training function of BP neural network}

This paper selects Levenberg-Marquardt algorithm as the learning algorithm. Compared with other learning algorithms,
Levenberg-Marquardt algorithm has the fastest convergence speed for the neural network which contains hundreds of weight values. In most cases, the mean square error is smaller when using the training function of the Levenbverg-Marquardt algorithm than that using other functions, and it can also reduce the probability of falling into local minma [9]. Levenberg-Marquardt algorithm (LM) can be amended by approximate Hessian matrix, which is given in Eq. (4).

$$
x(k+1)=x(k)-\left[\boldsymbol{J}^{T} \boldsymbol{J}+\mu \boldsymbol{I}\right]^{-1} \boldsymbol{J}^{T} \boldsymbol{e}
$$

where, $k$ is iteration times; $x(k)$ is the connection weight vector or threshold vector between the layers in the $k$ times iterations; $\boldsymbol{J}$ is Jacobi matrix; $\boldsymbol{J}^{T} \boldsymbol{J}$ indicates the Jacobi matrix which contains the first order derivative of the network error function to the weight and the threshold value.; $\mu \boldsymbol{I}$ is damping terms; $\boldsymbol{e}$ is error vector. When the coefficient $\mu$ is 0, Eq. (4) is the Newton method; when the value of the coefficient is large, Eq. (4) turns into smaller step gradient descent [10].

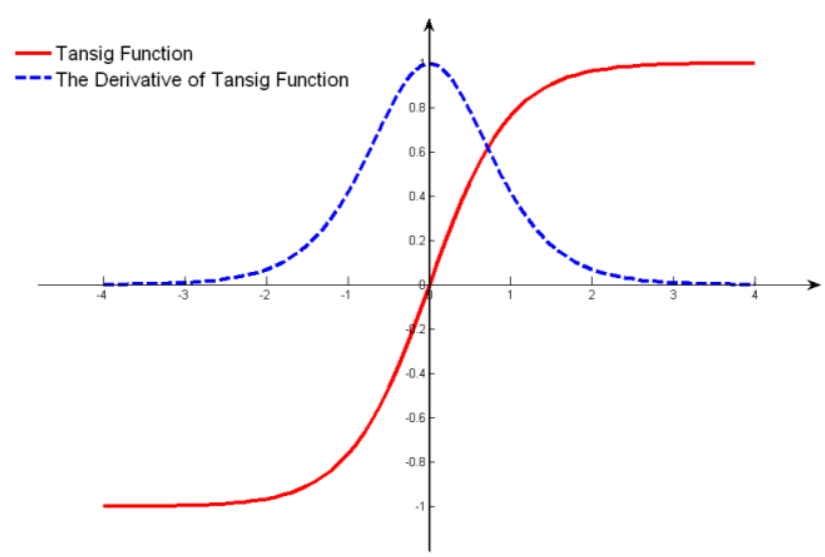

Fig.2. Tansig functional and its derivative value

2.2 The BP neural network optimized by the genetic algorithm

Genetic Algorithms, which simulates the genetic mechanisms and biological evolution in nature, is a parallel random search optimization method. It simulates the natural selection and the phenomenon of crossover and mutation, starting from initial population by random selection, crossover and mutation, resulting in a group of individuals better adapted to the environment. The population will gradually evolve into the optimization area of search space, and finally converge to the group individuals that are better adapted to their environment by reproduction and evolution, and then seeking the optimal solution [11].

The BP optimized by the genetic algorithm can be divided into three part, including determining the structure of $\mathrm{BP}$ neural network, optimizing the genetic algorithm and predicting BP neural network. The genetic algorithm has good global optimization ability. The BP neural network is optimized by the genetic algorithm in order to find the optimal 
initial value of BP neural network. Firstly, initial weights and thresholds are expressed by individual network, and the predicting errors of the BP neural network are regarded as the fitness of individuals. Secondly, the best individual is found by selection, crossover and mutation operation. The BP neural network has four input nodes, six output nodes and nine hidden layer nodes. Therefore, there are $4 \times 9+9 \times 6=90$ weights and $9+6=15$ threshold values, and the code length of genetic algorithm is $90+15=105$.

In genetic algorithm, fitness function is used to measure the quality of the individual to find the optimal solution in the optimization calculation, and it can be expressed by the reciprocal of the square sum of the data errors, as shown in $E q$. (5). [4].

$$
f(x)=\frac{1}{\sum_{i=1}^{n}\left[s_{i}(x)-s_{i}\right]^{2}}
$$

Where, $n$ is the number of output-layer nodes; $s_{i}(x)$ is the prediction output of the node; $S_{i}$ is the expection output of the node.

2.3 The BP neural network optimized by the particle swarm optimization

Particle Swarm Optimization is inspired by the group predatory behavior characteristics of birds and is used for solving problems. Each particle represents a potential solution of the problem, and each particle corresponds to a fitness value determined by the fitness function. The direction and distance of particle movement is determined by the velocity of the particles, which can be adjusted according to the moving experiences of itself and other particles. Therefore, the particles can search in a solution space which is fairly small with optimal solution.[12]

In each iteration process, particles update their speed and position by individual extreme and global extreme, as shown in $E q$. (6).

$$
\begin{aligned}
& V_{i d}^{k+1}=\omega V_{i d}^{k}+c_{1} r_{1}\left(P_{g d}^{k}-X_{i d}^{k}\right)+c_{2} r_{2}\left(P_{g d}^{k}-X_{i d}^{k}\right) \\
& X_{i d}^{k+1}=X_{i d}^{k}+V_{i d}^{k+1}
\end{aligned}
$$

Where, $\omega$ is the inertia weight; $d=(1,2, \ldots, D) ; i=(1$, $2, \ldots, n) ; k$ is iterations; $V_{\text {id }}$ is the velocity of the particles; $c_{1}$ and $c_{2}$ are nonnegative constants; $r_{1}$ and $r_{2}$ are random numbers in the interval $[0,1]$. [13]

The fitness function of the BP neural network optimized by the particle swarm optimization is shown in formula(11) [14].

$$
T=\frac{1}{N} \sum_{i=1}^{N} \sum_{j=1}^{m}\left(y_{j, i}^{d}-y_{j, i}\right)^{2}
$$

Where, $N$ is the total number of training samples; $y_{j, i}^{d}$ is the prediction value of the $\mathrm{j}$-th output node of the $\mathrm{i}$-th samples; $y_{j, i}$ is the expectations of the $\mathrm{j}$-th output node of the i-th samples; $m$ is the number of output neurons.

\section{DisPlacEMENT BACK ANALYSIS OF TUNNELS}

\subsection{The establishment of numerical model}

Numerical model is built based on No.3304 haulage gate in Dongtan Mine, China. The tunnels located in the $-660 \mathrm{~m}$ level. The tunnel was excavated in the coal seam along the floor, and the width and height are $5 \mathrm{~m}$ and $4 \mathrm{~m}$. The immediate roof of coal seam was siltstone and the main roof was middle-fine sandstone, while the immediate bottom was siltstone, and the main bottom was fine sandstone. Bolt-cable support system was adopted.. The simplified geometric model was established through the FLAC3D numerical simulation, which is shown in Fig.3.

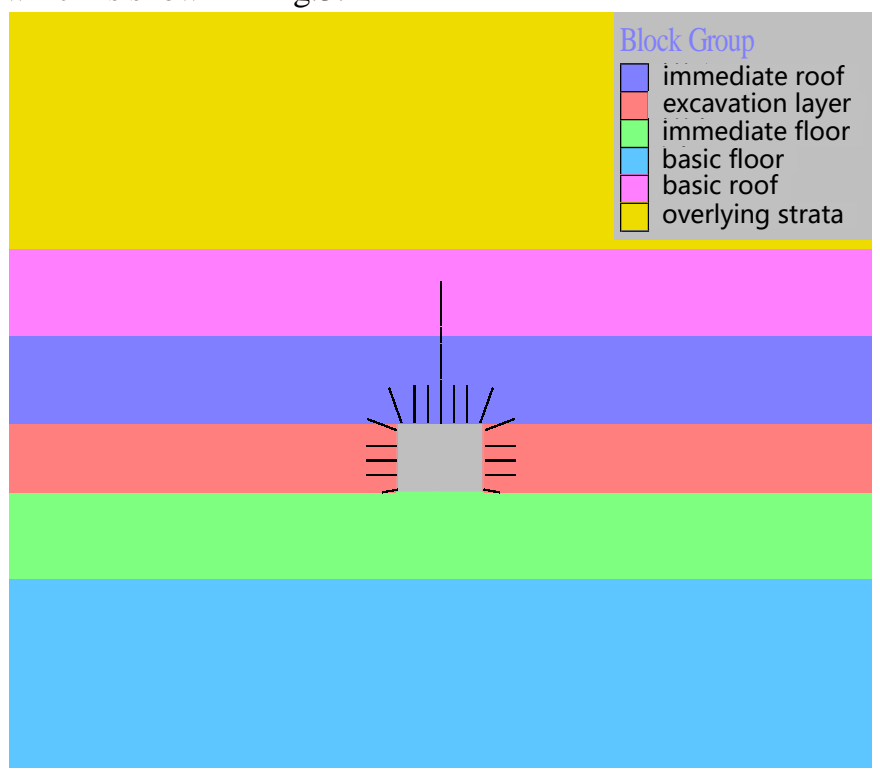

Fig.3. Simplified space geometry model

The width and height are $5 \mathrm{~m}$ and $4 \mathrm{~m}$ in the numerical model. The size of the model is set to be (length $\times$ width $\times$ height) $49 \times 50 \times 40 \mathrm{~m}$. The grids around the excavation tunnels are divided densely to improve the simulation precision. Displacement boundary condition is applied on the boundaries of the model (Itasca Consulting Group, Inc., 1997). X-velocity is set to be zero at both left and right boundaries. Y-velocity is set to zero at both front and back boundaries. X-velocity, $\mathrm{y}$-velocity and z-velocity are all set to be zero at the bottom boundary. The top boundary is free.

\subsection{Analysis of orthogonal experiments}

Different rock mass mechanical parameters have different effects on the displacement of the tunnels. The orthogonal experiments were designed and parameters, were chosen as experiment factors for analyzing the influence of rock mass mechanical parameters on the displacement of the tunnels[15]. According to the range of rock mechanics parameters, the $\mathrm{L}_{16}\left(4^{5}\right)$ orthogonal test table, which has four levels, was designed as the orthogonal test in this paper, as shown in Table 1 . 
TABLE 1. Factors of the orthogonal table and their levels

\begin{tabular}{llllll}
\hline Levels & $E / \mathrm{GPa}$ & $\mu$ & $c / \mathrm{MPa}$ & $\varphi /\left(^{\circ}\right)$ & $\gamma /\left(\mathrm{kN} / \mathrm{m}^{-3}\right)$ \\
\hline 1 & 2.0 & 0.1 & 1 & 10 & 20 \\
2 & 3.0 & 0.2 & 2 & 20 & 22 \\
3 & 4.0 & 0.3 & 3 & 30 & 24 \\
4 & 5.0 & 0.4 & 4 & 40 & 26 \\
\hline
\end{tabular}

The analysis results show that Young's modulus and Possion's ratio have great influence on the displacement of the tunnels. The deformation of the roof, floor and two side walls of the tunnels was monitored. For this part, the L25(56) orthogonal experiment, which has six experiment factors and five levels, was designed, as shown in table 2.

The deformation of tunnels were calculated, which was taken as the inputs of network training specimen. The three groups of the mechanical feature parameters of surrounding rock were used as outputs. Then, a unitary processing for the training samples was made, as shown in Table 3.

\subsection{Results of the back analysis validation}

The samples are trained with GA-BP and PSO-BP neural network using the neural network toolbox in MATLAB. The field data was selected as the test samples based on the field-datas of No. 3304 haulage tunnels in Dongtan Mine, China. The test samples were calculated by back analysis, which were taken as the trained neural network, and then the results of back analysis were calculated. The errors, the numerical values and test samples were compared and analyzed

TABLE 2. Factors of the orthogonal table and their levels

\begin{tabular}{cllllll}
\hline Levels & $E_{1} / \mathrm{GPa}$ & $\mu_{1}$ & $E_{2} / \mathrm{GPa}$ & $\mu_{2}$ & $E_{3} / \mathrm{GPa}$ & $\mu_{3}$ \\
\hline 1 & 1.0 & 0.1 & 1.0 & 0.1 & 1.0 & 0.1 \\
2 & 2.0 & 0.2 & 2.0 & 0.2 & 2.0 & 0.2 \\
3 & 3.0 & 0.3 & 3.0 & 0.3 & 3.0 & 0.3 \\
4 & 4.0 & 0.4 & 4.0 & 0.4 & 4.0 & 0.4 \\
5 & 5.0 & 0.45 & 5.0 & 0.45 & 5.0 & 0.45 \\
\hline
\end{tabular}

TABLE 3. The normalized mechanical parameters and the tunnels displacement

\begin{tabular}{ccccccccccc}
\hline No. & $E_{1}$ & $\mu_{1}$ & $E_{2}$ & $\mu_{2}$ & $E_{3}$ & $\mu_{3}$ & $X_{1}$ & $X_{2}$ & $X_{3}$ & $X_{4}$ \\
\hline 1 & -1 & -1 & -1 & -1 & -1 & -1 & 1 & 1 & 1 & -0.4285 \\
2 & -1 & -0.4285 & -0.5 & -0.4285 & -0.5 & 0.62222 & -0.01186 & -0.01105 & -0.2509 \\
3 & -1 & 0.14285 & 0 & 0.14285 & 0 & 0.14285 & 0.38311 & -0.3425 & -0.3424 & -0.6652 \\
4 & -1 & 0.71428 & 0.5 & 0.71428 & 0.5 & 0.71428 & 0.06431 & -0.5503 & -0.5543 & -0.8730 \\
5 & -1 & 1 & 1 & 1 & 1 & 1 & -0.1467 & -0.6766 & -0.6792 & -0.9817 \\
6 & -0.5 & 0.14285 & -1 & 0.71428 & -0.5 & 1 & -0.2731 & -0.0504 & -0.0507 & -0.56111 \\
7 & -0.5 & 0.71428 & -0.5 & 1 & 0 & -1 & -0.5203 & -0.6369 & -0.6377 & -0.5266 \\
8 & -0.5 & 1 & 0 & -1 & 0.5 & -0.4285 & -0.6639 & -0.6037 & -0.6042 & -0.7848 \\
9 & -0.5 & -1 & 0.5 & -0.4285 & 1 & 0.14285 & -0.3215 & -0.7866 & -0.7879 & -0.9502 \\
10 & -0.5 & -0.4285 & 1 & 0.14285 & -1 & 0.71428 & -0.2530 & -0.4693 & -0.4698 & 0.12123 \\
$\vdots$ & $\vdots$ & $\vdots$ & $\vdots$ & $\vdots$ & $\vdots$ & $\vdots$ & $\vdots$ & $\vdots$ & $\vdots$ \\
23 & 1 & -1 & 0 & 1 & -0.5 & 0.71428 & -0.8677 & -0.8505 & -0.853 & -0.5386 \\
24 & 1 & -0.4285 & 0.5 & -1 & 0 & 1 & -0.9392 & -0.8492 & -0.8504 & -0.8716 \\
25 & 1 & 0.14285 & 1 & -0.4285 & 0.5 & -1 & -1 & -0.9919 & -0.9924 & -0.7606 \\
\hline
\end{tabular}


TABLE 4. Back-analysis result of tunnels displacement

\begin{tabular}{cllllll}
\hline $\begin{array}{c}\text { Inversion } \\
\text { parameters }\end{array}$ & $\begin{array}{c}\mathrm{E} 1 / \\
\mathrm{GPa}\end{array}$ & $\mu 1$ & $\begin{array}{c}\mathrm{E} 2 / \\
\mathrm{GPa}\end{array}$ & $\mu 2$ & $\begin{array}{c}\mathrm{E} 3 / \\
\mathrm{GPa}\end{array}$ & $\mu 3$ \\
\hline $\mathrm{BP}$ & 2.633 & 0.352 & 1.258 & 0.306 & 3.251 & 0.396 \\
GA-BP & 2.761 & 0.367 & 1.236 & 0.315 & 2.918 & 0.364 \\
PSO-BP & 2.790 & 0.359 & 1.213 & 0.283 & 3.024 & 0.387 \\
\hline
\end{tabular}

TABLE 5. Comparison between actual displacement and calculated displacement

\begin{tabular}{|c|c|c|c|c|c|}
\hline \multicolumn{2}{|c|}{ Measuring point } & tunnel's roof & tunnel's left side & tunnel's right side & tunnel's floor \\
\hline \multicolumn{2}{|c|}{ Actual displacement value $/ \mathrm{mm}$} & 128 & 157 & 159 & 72 \\
\hline \multirow{6}{*}{$\begin{array}{c}\text { Calculated } \\
\text { displacement value } \\
/ \mathrm{mm}\end{array}$} & $\mathrm{BP}$ & 133.2 & 151.7 & 151.1 & 65.1 \\
\hline & error $1 \%$ & 4.06 & 3.37 & 4.97 & 9.58 \\
\hline & GA-BP & 126.5 & 153.6 & 153.7 & 75.9 \\
\hline & error $1 \%$ & 0.078 & 2.17 & 3.33 & 6.81 \\
\hline & PSO-BP & 129.5 & 160.2 & 160.1 & 69.2 \\
\hline & error $1 \%$ & 1.17 & 2.04 & 0.69 & 3.89 \\
\hline
\end{tabular}

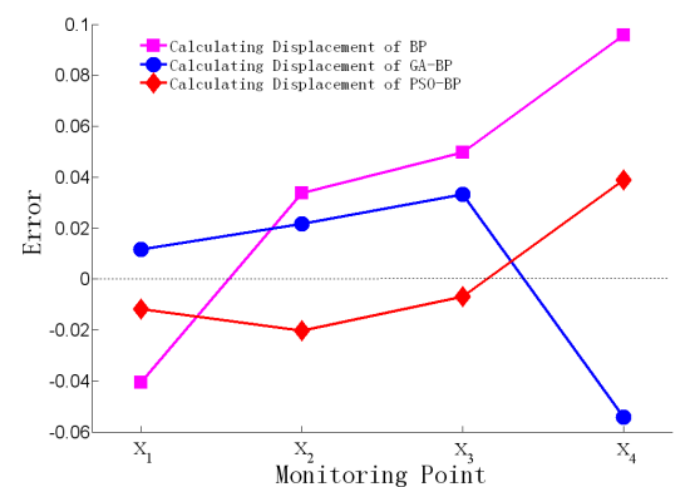

Fig.4. Error curve of the calculated displacement

\section{CONCLUSION}

The BP neural network optimized by the genetic algorithm and particle swarm optimization has global searching ability, and the back analysis effect is better than the BP neural network without optimization.

The GA-BP and the PSO-BP neural network has better inversion accuracy, while the PSO-BP neural network has quicker convergence rate and stable inversion results. However, these three neural networks have some errors for the floor displacement calculation and it needs to be optimized further.
The prediction accuracy of the three neural networks was more than $90 \%$, which indicates that using artificial neural network to back analyze the deformation of the tunnels is a good option, and the back-analysis mechanical parameters of the surrounding rock have a certain credibility. This research can provide a reference for the tunnels support design.

\section{ACKNOWLEDGEMENT}

This work is supported by National Natural Science Foundation of China (51274133, 51474137), Shandong Province Science and Technology Development Plan Item (2014GSF120002), and Tai'shan Scholar Engineering Construction Fund of Shandong Province of China.

\section{REFERENCES}

[1] Tan Yun-Liang, Ning Jian-Guo, Zhao Tong-Bin, Zang Chuan-Wei. Failure and Control of Surrounding Rock in Deep tunnels [M], China Coal Industry Publishing House, 2011.

[2] Li Yong-Jian, Li Zi-Sheng. Review of Intelligent Back Exercise of Displacement Back Analysis in Geo-technical Engineering [J], Chinese Journal of Underground Space and Engineering, 2004, 24(1):84-88.

[3] Tan Yun-Liang, Wang Yong-Jia. Neural Network Model of Cluster Analysis for the Classification Indexes of Gate [J], Chinese Journal of Rock Mechanics and Engineering, 1995(2): 139-144.

[4] Wu Jian-Tong, Wang Jian-Hua. Neural Network Technology and Its Application [M], Harbin Engineering University Press, 1998.

[5] Zhao Tong-Bin, Tan Yun-Liang, Liu Chuan-Xiao. Research on 
back-analysis of tunnels displacement based on genetic algorithms [J], Rock and Soil Mechanics, 2004, 25(S1): 107-109.

[6] WANG Xue-Dong, LI Guang-Jie, YOU Bing, QIN Sheng-Wu, PENG Shuai-Ying. tunnels Displacement Back Analysis Based on BP Neural Network Optimized by Particle Swarm [J], Journal of China Coal Society, 2012, (S1):38-42.

[7] Tan Yun-Liang, Sun Zhong-Hui, Du Xue-Dong. A Wavelet Neural Network Predicting Model for Acoustic Emission Time Series of Rock Burst [J], Chinese Journal of Rock Mechanics and Engineering, 2000, 19(S1): 1034-1036.

[8] Hecht Nielsen Robert. Application of Counter propagation Networks [J], Neural Networks, 1988, 1(2): 131-139.

[9] Zhou Kai-Li, Kang Yao-Hong. Neural Network Model and MATLAB programming [M], Tsinghua University Press, 2005.
[10] Martin T Hagan, Howard B Demuth, Mark H Beale. Neural Network Design [M]. Martin Hagan, 2014.

[11] Wang Xiao-Ping, Cao Li-Ming. Genetic algorithm theory, application and software implementation [M], Xi'an Jiaotong University Press, 2002.

[12] Yang Wei, Li Qi-Qiang. Survey on Particle Swarm Optimization Algorithm [J], Engineering Science, 2004, 6(5): 87-94.

[13] Liang Jun, Cheng Can. Modified Particle Swarm Optimization Algorithm [J], Computer Engineering and Design, 2008, 29(11): 2893-2896.

[14] Zhao Tong-Bin. Research on back-analysis of tunnels displacement based on genetic algorithms [D], Shandong University of Science And Technology, 2004

[15] Zhao Tong-Bin, Tan Yun-Liang, Zhang Yu-Ming, Shi Long-Qing. Inevitability Evaluation of Back-Analysis of tunnels Displacement [J], Journal of Mining \& Safety Engineering, 2006, 23(2): 224-227. 